\title{
Longitudinal profiles, fluctuations and correlations of electromagnetic cascades produced by 100-3500 MeV gamma quanta in heavy amorphous media
}

\author{
Bronisław Słowiński \\ National Centre for Nuclear Research, \\ ul. Andrzeja Soltana 7 05-400 Otwock, Poland \\ E-mail: slowbeif.pw.edu.pl
}

\section{Przemysław Duda}

Faculty of Physics, Warsaw University of Technology

ul. Koszykowa 75 00-662 Warszawa

E-mail: duda@if.pw.edu.pl

\section{Dariusz Mączka}

National Centre for Nuclear Research,

ul. Andrzeja Soltana 7 05-400 Otwock, Poland

E-mail: dariusz.maczka@ncbj.gov.pl

\section{Jacek Bzdak}

Faculty of Physics, Warsaw University of Technology ul. Koszykowa 75 00-662 Warszawa

E-mail: bzdakeif.pw.edu.pl

\begin{abstract}
The results of investigation of average longitudinal profiles, fluctuations and correlations of electromagnetic cascades created in dense amorphous media (liquid xenon, $\mathrm{PWO}, \mathrm{CdWO}_{4}$, $\mathrm{GaAs}, \mathrm{NaI}, \mathrm{Pb}$, lead glass and $\mathrm{BGO}$ ) by gamma quanta of energy $E_{\gamma}=100 \div 3500 \mathrm{MeV}$ at four different cut-off energies $E_{c o}$ of cascade's electrons and positrons $(0.6,1.2,2.0$ and $3.0 \mathrm{MeV})$ are reported. The work has been performed using EGS4 [3] and GEANT modelling codes [4]. The modelled results are compared with experimental data for liquid xenon [2].
\end{abstract}

XXI International Baldin Seminar on High Energy Physics Problems September 10-15, 2012

JINR, Dubna, Russia 


\section{INTRODUCTION}

Presently in use is practically the simplest and rough description of the main features of electromagnetic cascades (ECs or showers created in dense amorphous materials (DAMs) by high energy gamma quanta (GQ): longitudinal profiles (LPs) and several integral formulas describing the longitudinal energy deposition in ECs as estimations of energy resolution of photodetectors (for example, [1,2]). So, it is commonly assumed that the longitudinal distributions of energy deposition (or profiles) of ECs initiated in DAMs can be approximated satisfactorily by a gamma type function (for example, [1,2]). Nevertheless, such a simple model is not too adequate, especially at large depths $t$ and at the very beginning of the cascade [2]. Furthermore, since the longitudinal dimensions of ECs increase with primary photons energy $E_{\gamma}$ as $\ln E_{\gamma}$ then at dimensions of electromagnetic calorimeters limited, usually to 20-25 radiation lengths (r.l.), it is also important to have information about longitudinal fluctuations in ECs. Moreover, in order to reconstruct as far as possible a real ECs picture, and so to estimate with acceptable accuracy the primary gamma quanta energy, of particular interest is also the knowledge on correlations in longitudinal EC development, what is of prime importance for segmented gamma detectors.

In the work we study the average longitudinal profiles, fluctuations and correlations of ECs created in eight most popular dense amorphous media: liquid xenon, $\mathrm{PWO}, \mathrm{CdWO}_{4}, \mathrm{GaAs}$, $\mathrm{NaI}, \mathrm{Pb}$, lead glass and BGO by gamma quanta of energy $E_{\gamma}=100 \div 3500 \mathrm{MeV}$ at four different cut-off energies $E_{c o}$ of electrons and positrons (later on: electrons): 0.6, 1.2, 2.0 and 3.0 MeV. The fluctuations have been investigated as a distribution of the shower depth, up to which a part A of energy $E_{\gamma}$, simply called as a threshold energy (TE), was deposited. Estimated are also correlations in longitudinal energy deposition of ECs. The work has been performed using EGS4 [3] and GEANT modeling codes [4]. For every set of parameters: $E_{\gamma}, E_{c o}$ and material 20000 events (histories) have been modeled. The obtained results are compared with experimental data for liquid xenon [2].

\section{AVERAGE LONGITUDINAL PROFILES}

The results of modeling longitudinal ECs profiles have been fitted with the function,

$$
(-d E / d t)=\alpha_{t}\left(t-\varepsilon_{t}\right)^{\beta_{t}} \exp \left(-\gamma_{t} t^{\delta_{t}}\right)
$$

here $\alpha_{t}, \beta_{t}, \delta_{t}, \gamma_{t}, \varepsilon_{t}$ are the fit parameters depending on cut-off energy $E_{c o}$ of cascade particles and material properties. They are determined as a result of statistic approximation to calculated data. Fig. 1 shows as an example the LP in the form of ionization losses of electrons of EC created in BGO by GQ of energy $1625 \mathrm{MeV}$ at $E_{c o}=1.2 \mathrm{MeV}$. The solid curve represents the fitted function (1) with values of parameters shown as inlet.

In Table 1 collected are numerical values of parameters of function (1) for two materials: liquid Xe doped with $0.5 \% \mathrm{C}_{2} \mathrm{H}_{4}$ by weight and PWO at $E_{c o}=1.2 \mathrm{MeV}$. 
Next, the relationship between the values of parameters $\beta_{t}, \delta_{t}, \gamma_{t}$, and $\varepsilon_{t}$, and the initial GQ energy and media properties was investigated. To characterize different media the coefficient $\mathrm{W}$ was defined as $(\mathrm{A} / \mathrm{Z}) \rho$ where $\mathrm{A}$ is the mean mass number of components of the material, $\mathrm{Z}$ is the mean atomic number of its elements, $\rho-$ material density. Below we depicted the dependences for each of these parameters $\beta_{t}, \delta_{t}, \gamma_{t}$, and $\varepsilon_{t}$ in turn, on $E_{\gamma}, E_{c o}$ and W.

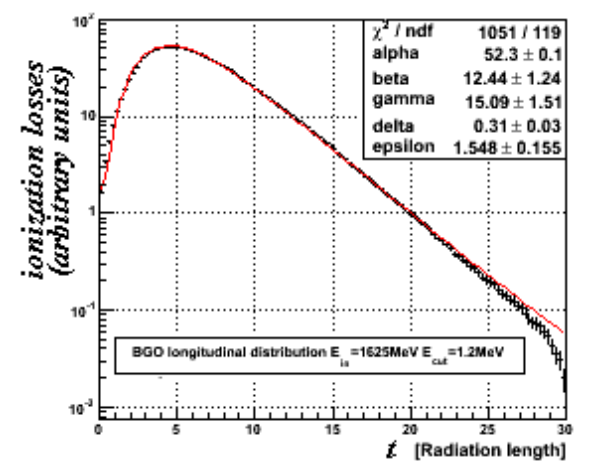

Fig.1. Longitudinal profile of electromagnetic cascades created in BGO by gamma quanta of energy $E_{\gamma}=1625 \mathrm{MeV}$ when $E_{c o}=1.2 \mathrm{MeV}$. Solid line represents the fitted function (1) with values of parameters shown in the inlet.

\begin{tabular}{|l|ccccccccccc|}
\hline material & $\mathrm{E}_{\boldsymbol{\gamma}}$ & $\mathrm{E}_{\mathbf{c o}}$ & $\beta_{\mathbf{t}}$ & $\boldsymbol{\Delta} \boldsymbol{\beta}_{\mathbf{t}}$ & $\boldsymbol{\gamma}_{\mathbf{t}}$ & $\Delta \boldsymbol{\gamma}_{\mathbf{t}}$ & $\delta_{\mathbf{t}}$ & $\Delta \delta_{\mathbf{t}}$ & $\boldsymbol{\varepsilon}_{\mathbf{t}}$ & $\boldsymbol{\Delta} \boldsymbol{\varepsilon}_{\mathbf{t}}$ & $\boldsymbol{x}^{\mathbf{2}} / \mathbf{n d f}$ \\
\hline & 210 & & 4.0 & 0.4 & 3.6 & 0.3 & 0.42 & 0.04 & 0.7 & 0.1 & $144 / 119$ \\
& 555 & & 8.1 & 0.8 & 8.9 & 0.8 & 0.34 & 0.03 & 1.2 & 0.1 & $240 / 119$ \\
$\mathrm{Xe}+\mathrm{C}_{2} \mathrm{H}_{\mathbf{4}}$ & 875 & 1.2 & 10.0 & 1.0 & 11.6 & 1.1 & 0.32 & 0.03 & 1.3 & 0.1 & $456 / 119$ \\
$\mathrm{~W}=$ & 1625 & & 12.5 & 1.2 & 15.2 & 1.5 & 0.31 & 0.03 & 1.5 & 0.1 & $908 / 119$ \\
$2.2 \mathrm{~g} / \mathrm{cm}^{3}$ & 2375 & & 14.0 & 1.4 & 17.4 & 1.7 & 0.30 & 0.03 & 1.6 & 0.1 & $1289 / 119$ \\
& 3125 & & 15.1 & 1.5 & 19.1 & 1.9 & 0.29 & 0.03 & 1.6 & 0.1 & $1712 / 119$ \\
\hline & 210 & & 4.3 & 0.4 & 4.1 & 0.4 & 0.40 & 0.04 & 0.8 & 0.1 & $374 / 119$ \\
$\mathrm{PWO}$ & 555 & & 8.3 & 0.8 & 9.2 & 0.9 & 0.34 & 0.03 & 1.3 & 0.1 & $313 / 119$ \\
$\mathrm{~W}=$ & 875 & 1.2 & 10.1 & 1.0 & 11.7 & 1.1 & 0.32 & 0.03 & 1.4 & 0.1 & $448 / 119$ \\
$20.1 \mathrm{~g} / \mathrm{cm}^{3}$ & 1625 & & 12.6 & 1.2 & 15.4 & 1.5 & 0.30 & 0.03 & 1.5 & 0.1 & $969 / 119$ \\
& 2375 & & 14.1 & 1.4 & 17.8 & 1.7 & 0.29 & 0.03 & 1.6 & 0.1 & $1488 / 119$ \\
& 3125 & & 15.3 & 1.5 & 19.5 & 1.9 & 0.29 & 0.02 & 1.7 & 0.1 & $1923 / 119$ \\
\hline
\end{tabular}

\section{Table 1}

Numerical values of parameters $\beta_{t}, \delta_{t}, \gamma_{t}, \varepsilon_{t}$ of the function (1) approximating longitudinal profiles of showers created by gamma quanta of energy $\mathrm{E}_{\gamma}(\mathrm{MeV})$ in liquid Xe doped with $0.5 \%$ $\mathrm{C}_{2} \mathrm{H}_{4}$ by weight and PWO at $E_{c o}=1.2 \mathrm{MeV}$. 


\subsection{Parameter $\beta_{t}$}

Fig. 2. shows the dependence of parameter $\boldsymbol{\beta}_{\mathfrak{t}}$ of function (1) on the primary GQ energy $\mathrm{E}_{\gamma}$, cut-off energy $\mathrm{E}_{\mathrm{co}}$ and absorber. This dependence may be approximated by a function:

$$
\beta_{t}\left(E_{\gamma}\right)=a_{1} \cdot E_{\gamma}^{b_{1}}+c_{1}
$$

where $a_{l}, b_{1}$ and $c_{l}$ are parameters.

One can notice that the parameter $\beta_{\mathrm{t}}$ does not depend within statistical errors both from cut-off energy in the investigated interval $0.6-3.0 \mathrm{MeV}$ and slightly depends on absorber property.

\subsection{Parameter $\gamma_{\mathrm{t}}$}

Similarly as above, Fig. 3 shows the dependence of parameter $\gamma_{\mathbf{t}}$ of function (1) on primary GQ energy $E_{\gamma}$, cut-off energy $E_{c o}$ and absorber property. This dependence may be approximated by the function:

$$
\gamma_{t}\left(E_{\gamma}\right)=a_{2} \cdot E_{\gamma}^{b_{2}}+c_{2}
$$

where $a_{2}, b_{2}$ and $c_{2}$ are parameters to be fitted.

\subsection{Parameter $\delta_{\mathrm{t}}$}

In a like manner as above Fig.4. demonstrates the dependence of parameter $\boldsymbol{\delta}_{\mathbf{t}}$ of function (1) on energy $E_{\gamma}$, cut-off energy $E_{c o}$ and absorber property. This dependence has been approximated by the function:

$$
\delta_{t}\left(E_{\gamma}\right)=a_{3} \cdot E_{\gamma}^{-b_{3}}+c_{3}
$$

where $a_{3}, b_{3}$ and $c_{3}$ are parameters.

\subsection{Parameter $\varepsilon_{\mathrm{t}}$}

The primary GQ energy dependence of parameter $\boldsymbol{\varepsilon}_{\mathbf{t}}$ at various cut-off energies and in different materials is depicted in Fig.5.

Hence one can conclude that all investigated parameters $\beta_{t}, \delta_{t}, \gamma_{t}$ and $\varepsilon_{t}$ of the function (1) describing the longitudinal profiles of ECs initiated in dense amorphous materials by GQ of energy $E_{\gamma}=100 \div 3500 \mathrm{MeV}$ do not depend within statistical errors on the cut-off energy in the range $E_{c o}=0.6 \div 3.0 \mathrm{MeV}$ and slightly depend on material properties, and can be approximated by simple functions of $E_{\gamma}(2-4)$. 

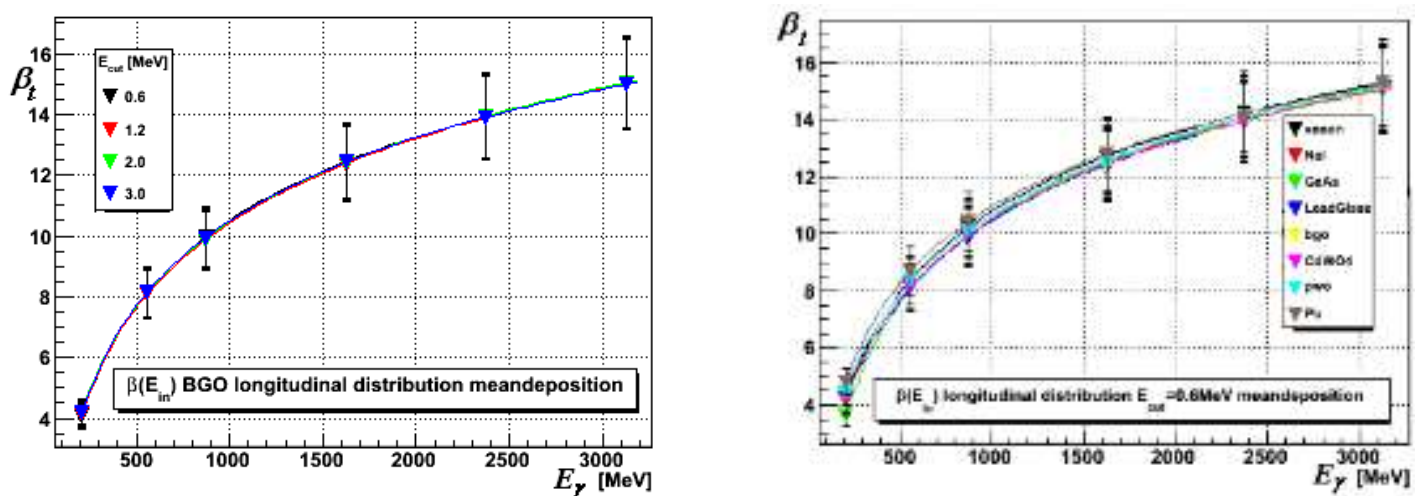

Fig.2. Dependence of the parameter $\boldsymbol{\beta}_{t}$ of function (1) on the primary energy $E_{\gamma}$ producing showers in BGO and registered at different cut-off energy $E_{c o}$ (left) and in different absorbers (right) at $E_{c o}=0.6 \mathrm{MeV}$. Solid lines represent the fitting function (2).
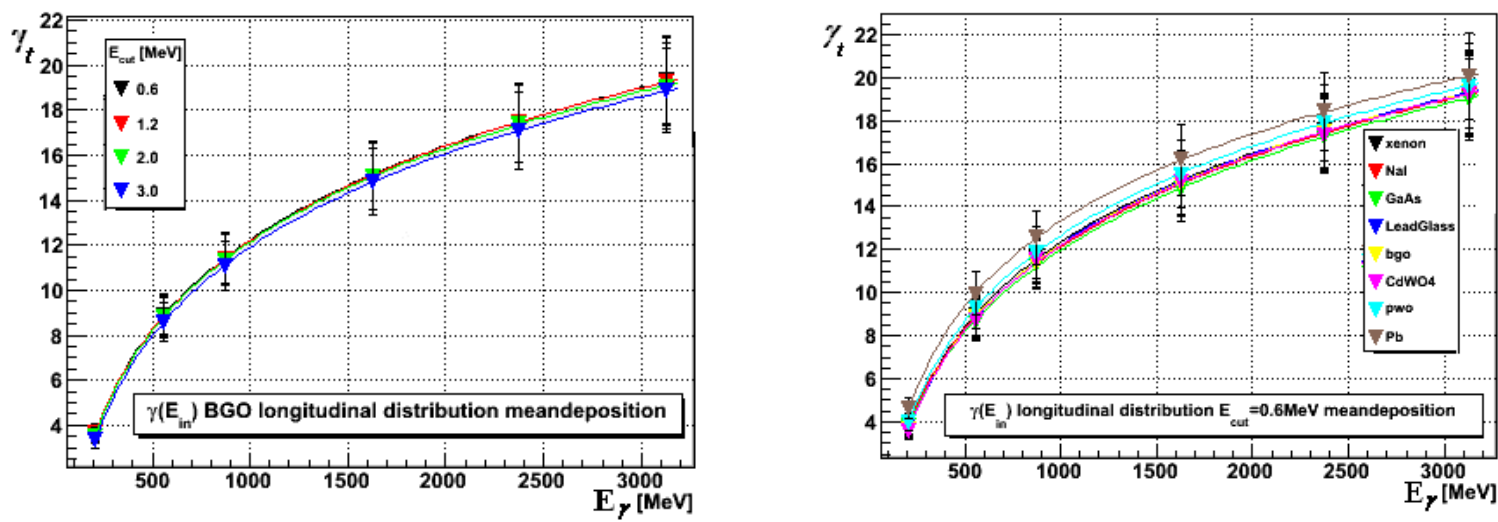

Fig.3. The same as Fig.2. but for parameter $\gamma_{t}$. Solid lines represent the approximating function (3).
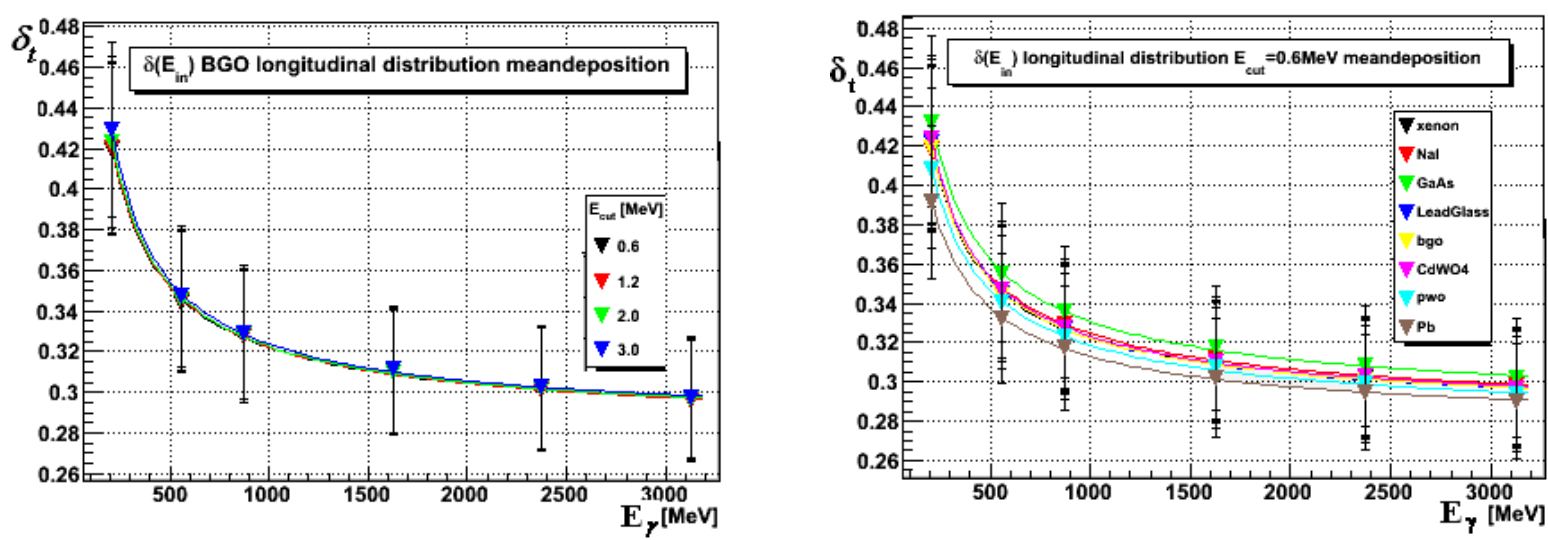

Fig.4. The same as Fig.3 but for parameter $\boldsymbol{\delta}_{t}$. Solid lines represent approximating function (4). 

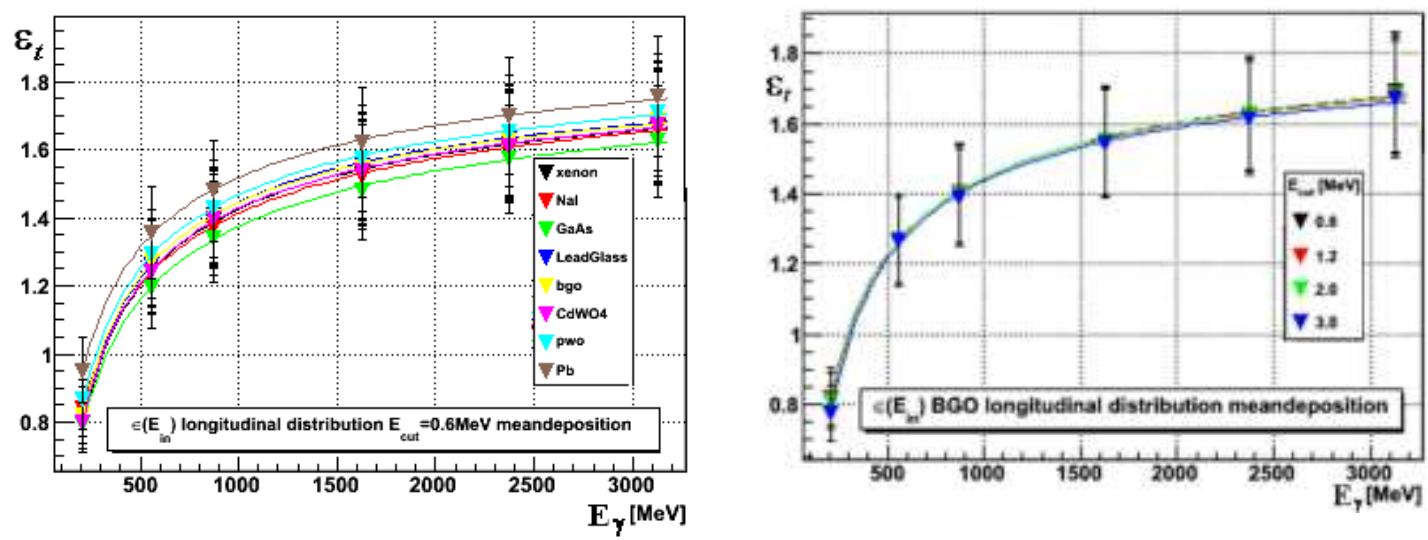

Fig.5. The same as Fig.3 but for parameter $\boldsymbol{\varepsilon}_{t}$.
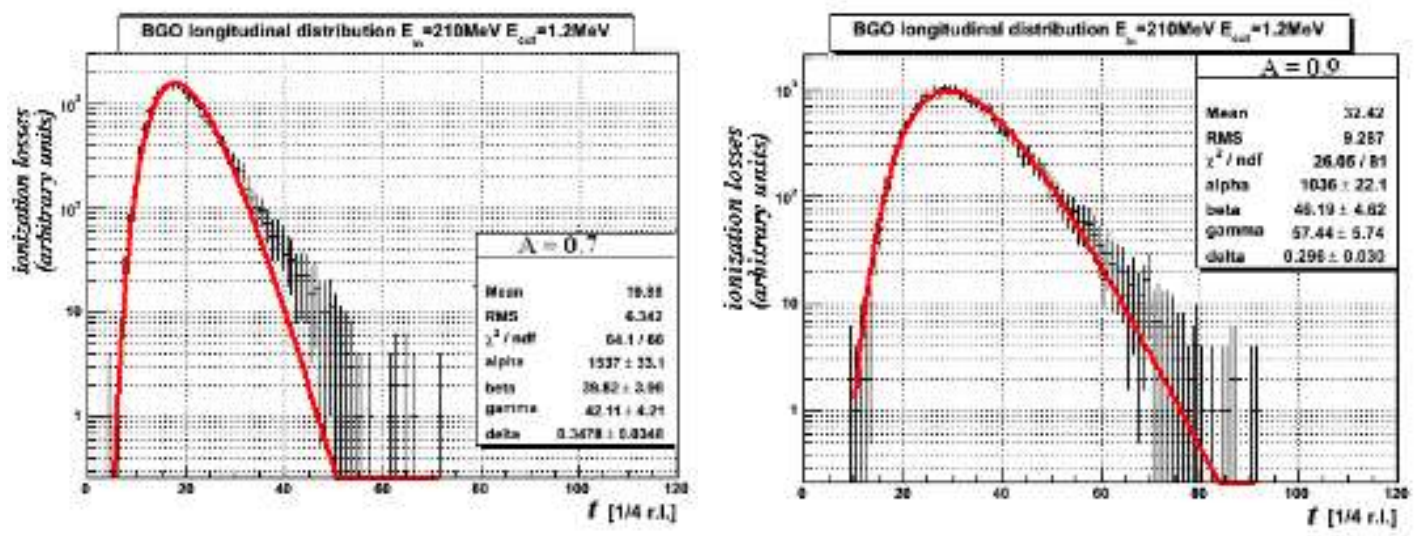

Fig.6. Ddistributions of the shower depth $t_{A}$ at which fixed parts $\mathrm{A}=0.7$ (left) and 0.9 (right) of the total energy $\mathrm{E}_{\gamma}$ is released when the cascade is initiated in BGO by gamma quanta of energy $E_{\gamma}=210 \mathrm{MeV}$ and detected at $E_{c o}=1.2 \mathrm{MeV}$. Solid line represents the fitting function (5).

\begin{tabular}{|c|c|c|c|c|c|c|c|c|c|}
\hline material & $E_{y}, M e V$ & $\mathrm{E}_{\text {co }}, \mathrm{MeV}$ & $\beta$ & $\Delta \beta$ & $y$ & $\Delta y$ & 8 & $\Delta 8$ & $x^{2} / \mathbf{n d f}$ \\
\hline \multirow{6}{*}{$\mathrm{BGO}$} & 210 & \multirow{6}{*}{1.2} & 40 & 4 & 42 & 4 & 0.35 & 0.04 & $69 / 68$ \\
\hline & 555 & & 73 & 7 & 85 & 8 & 0.32 & 0.03 & $53 / 67$ \\
\hline & 875 & & 92 & 9 & 111 & 11 & 0.30 & 0.03 & $56 / 65$ \\
\hline & 1625 & & 143 & 14 & 179 & 17 & 0.30 & 0.03 & $102 / 60$ \\
\hline & 2375 & & 149 & 15 & 191 & 19 & 0.29 & 0.03 & $65 / 58$ \\
\hline & 3125 & & 158 & 15 & 206 & 20 & 0.28 & 0.03 & $134 / 67$ \\
\hline \multirow{6}{*}{$\begin{array}{l}\text { lead } \\
\text { glass }\end{array}$} & 210 & \multirow{6}{*}{1.2} & 30 & 3 & 32 & 3 & 0.35 & 0.04 & $57 / 85$ \\
\hline & 555 & & 67 & 6 & 78 & 7 & 0.32 & 0.03 & $53 / 55$ \\
\hline & 875 & & 96 & 9 & 116 & 11 & 0.31 & 0.03 & $71 / 58$ \\
\hline & 1625 & & 128 & 12 & 161 & 16 & 0.29 & 0.03 & $74 / 60$ \\
\hline & 2375 & & 155 & 15 & 199 & 19 & 0.29 & 0.03 & $81 / 58$ \\
\hline & 3125 & & 160 & 16 & 208 & 20 & 0.28 & 0.03 & $101 / 64$ \\
\hline
\end{tabular}

Table 3. Numerical values of parameters $\beta, \gamma$ and $\delta$ of function (5) for $\mathrm{A}=0.7, E_{\gamma}=210 \mathrm{MeV}$, $E_{c o}=1.2 \mathrm{MeV}$, and two materials: $\mathrm{BGO}$ and lead glass. 


\section{LONGITUDINAL FLUCTUATIONS}

According to [2] we determine the fluctuations in ECs as a distribution of the shower depth $t_{\mathrm{A}}$ that starts at the shower beginning, up to the depth value where an amount $\mathrm{A}$ of total shower energy $E_{\gamma}$ was deposited. Three values of A having practical meaning were considered: $0.5,0.7$ and 0.9 .

Fig.6. illustrates, as an example, a distribution of the shower depth $t_{A}$ at which fixed parts $\mathrm{A}=0.7$ and 0.9 of the total cascade energy is released when the cascade is initiated in BGO by gamma quanta of energy $E_{\gamma}=210 \mathrm{MeV}$ and detected at $E_{c o}=1.2 \mathrm{MeV}$.

It turned out that all such distributions may be satisfactorily enough approximated by the function:

$$
P\left(t_{A}\right)=\alpha t_{A}^{\beta} \exp \left(-\gamma t_{A}^{\delta}\right)
$$

where numerical values of parameters $\beta, \gamma$ and $\delta$ have been determined as a fit to modeled results. In Table 3 given are numerical values of parameters $\beta, \gamma$ and $\delta$ for $\mathrm{A}=0.7, E_{\gamma}=210 \mathrm{MeV}, E_{c o}=1.2 \mathrm{MeV}$, and two materials: $\mathrm{BGO}$ and lead glass.

Fig.7. depicts the dependences of all these parameters on $E_{\gamma}$ and $E_{c o}$ for BGO. The $E_{\gamma}$ dependence may be parameterized by the following function

$$
p\left(E_{\gamma}\right)=a_{p} \cdot E_{\gamma}^{b_{p}}+c_{p}
$$

where $a, b$ and $c$ are constants for each parameter $p: \beta, \gamma$ and $\delta$.

\section{LONGITUDINAL CORRELATIONS}

Longitudinal dimensions of ECs increase with increasing energy of primary GQs and starting at some value which depends on stopping properties of an absorber they exceed the absorber thickness. Then the problem arise whether is it possible and to what extent to reconstruct the total energy of the primary GQs creating such cut off showers. This problem may be solved within the practical level when the EC energy is released inside an absorber of layered structure along the EC axis or is observed visually in track's detectors. As has been showed experimentally for liquid xenon [3] when analyzing depth distributions of the fraction $<\mathrm{A}>$ of ionization losses in showers produced by GQ of energy $E_{\gamma}=100 \div 3500 \mathrm{MeV}$ there exist short-range correlations of the longitudinal energy release in the vicinity of the shower maximum. For quantitative characteristics of these correlations we used convention definition of the correlation coefficient as

$$
r_{i j}=\operatorname{cov}\left(X_{i}, X_{j}\right) /\left[\sigma\left(X_{i}\right) \sigma\left(X_{j}\right)\right],
$$

where $X_{i}=\Delta<A>/ \Delta t_{i}$ is the slope of the random function at the depth $t_{i}$ of the absorber where at which on the average $i / 10$ of the total cascade energy is absorbed $(I=2,3, \ldots, 10)$, and the thickness of the layer $\Delta t_{i}=t_{i}-t_{i-1}$ is taken such that on the average a part $\Delta\langle A\rangle=0.1$ of this 

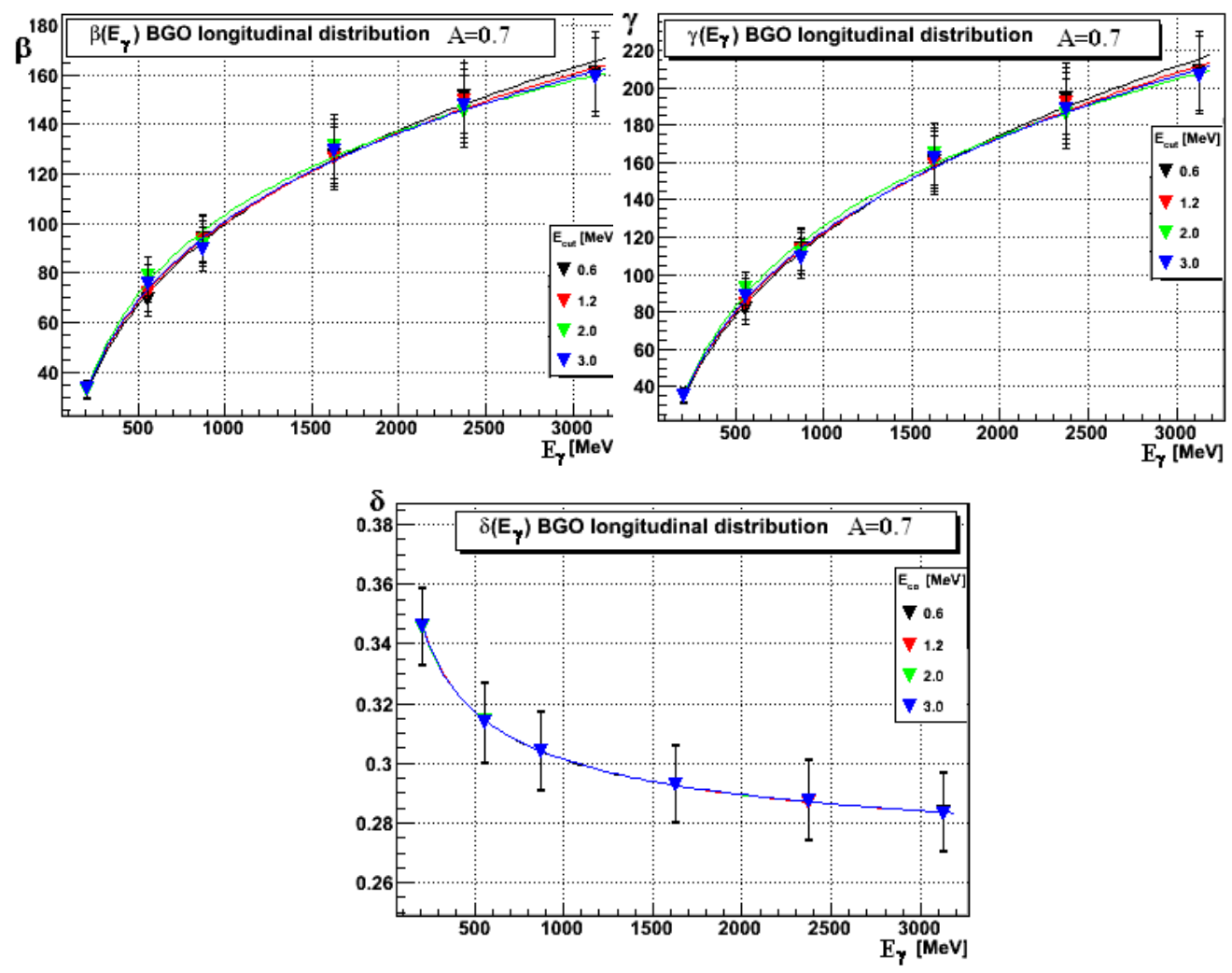

Fig.7. Dependence on energy $E_{\gamma}$ of parameters $\beta, \gamma$ and $\delta$ of function (6) for BGO at different $E_{c o}$.
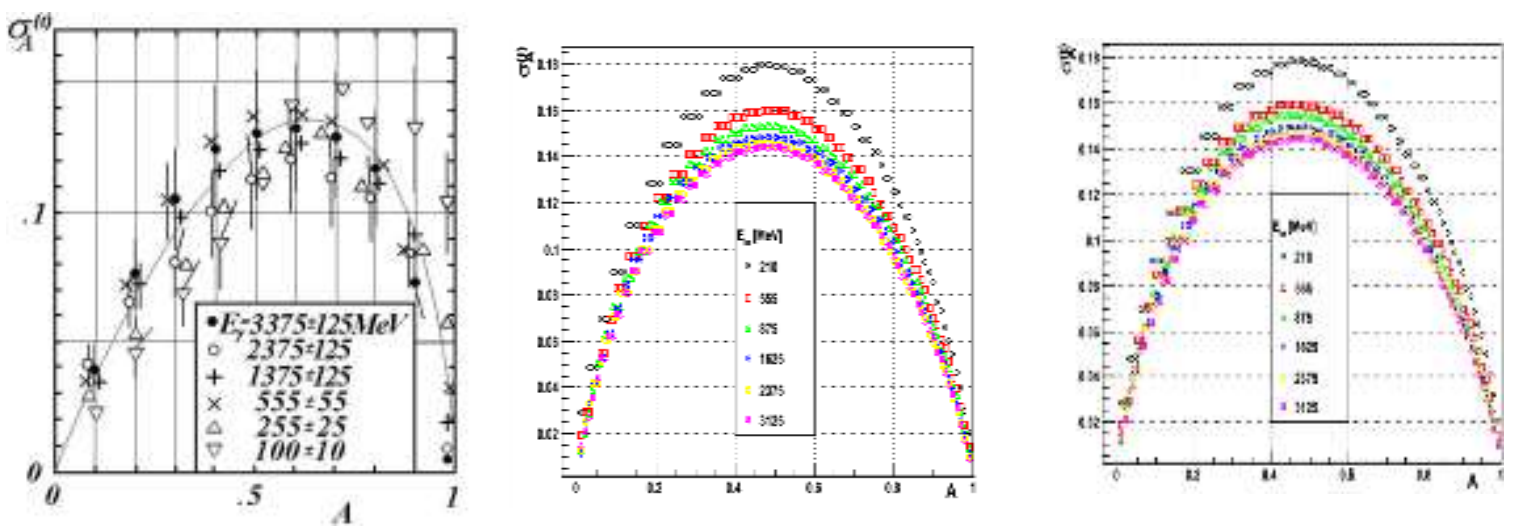

Fig.8. $\boldsymbol{A}$ dependence of the rms deviation $\sigma_{A}$ from the average fraction $\boldsymbol{A}$ of ionization losses for energy deposited along the axis of showers created in liquid xenon by GQs of energy $E_{\dot{\gamma}}$ left - experiment [2], at the center - calculation according EGS code [3], right - calculation using GEANT code [4]. 
energy is absorbed in it $(j>i)$. As an example in Tables 4 and 5 given are our modeled values of $r_{i j}$ for ECs created in liquid xenon by GQs of energy $555 \mathrm{MeV}$ and $1650 \mathrm{MeV}$ at $E_{c o}=1 \mathrm{MeV}$.

One can notice that there exist practically meaningful at least short range correlations among layers of energy losses in ECs providing a possibility to reconstruct a missing part of the cut off EC energy, and this possibility is better when the primary GQ energy is higher. Moreover, maximum value of rij is reached around the EC maximum.

\section{COMPARISON WITH EXPERIMENT}

We compared our model consisting in formulas (1) $\div(8)$ and constants $a_{p}, b_{p}$ and $c_{p}$ with available experimental data. It is worthy to be mentioned that the most exhaustive and various experimental information on ECs initiated in liquid xenon by GQs of energy $100 \div 3500 \mathrm{MeV}$ [2] has been obtained using the 180-liter Xenon Bubble Chamber (180XeBC) of ITEP, Moscow [5], although the accuracy of experimental data is rather on the quantitative level. In Fig.8 we demonstrate a comparison of our modeled results with experimental data from $180 \mathrm{XeBC}$ concerning longitudinal fluctuations.

Confrontation of modeled results on the correlation coefficient rij with the experiment from $180 \mathrm{XeBC}$ [2] displays similar tendency but absolute values of modeled $r_{i j}$ are systematically shifted to be higher for all GQ energy by about $10 \%$.

\section{CONCLUSION}

We have investigated using EGS [3] and GEANT codes [4] the essential features of electromagnetic cascades induced in dense amorphous media by gamma quanta of intermediate energy region $E_{\gamma}=100 \div 3500 \mathrm{MeV}$ : longitudinal profiles and longitudinal fluctuations of energy deposition. All these characteristics have been described as simple functions of primary GQ energy with parameters practically insensitive to cut-off energy within the interval $0.6 \div 3.0 \mathrm{MeV}$ of cascade electrons and slightly depending on material properties. Moreover, studied are also correlations in longitudinal development of cascades which may be helpful for reconstruction of the total shape of cascades registered in detectors of limited sensitive volume. The results have been confronted with available experimental data [2] showing acceptable agreement. In the case of correlation the agreement is on the level of general behavior but farther investigation should be done. Our model of electromagnetic cascades in the form of a set of simple formulas may be quite suitable both for electromagnetic calorimeters such as PANDA and for construction of radiation shielding. 


\begin{tabular}{|l|l|l|l|l|l|l|l|l|}
\hline $\mathrm{i} / \mathrm{j}$ & 3 & 4 & 5 & 6 & 7 & 8 & 9 & 10 \\
\hline 2 & $0.38 \pm 0.03$ & $0.16 \pm 0.03$ & $0.08 \pm 0.03$ & $0.04 \pm 0.03$ & $0.00 \pm 0.03$ & $-0.01 \pm 0.03$ & $-0.01 \pm 0.03$ & $-0.02 \pm 0.03$ \\
\hline 3 & $0.42 \pm 0.03$ & $0.19 \pm 0.03$ & $0.08 \pm 0.03$ & $0.03 \pm 0.03$ & $0.00 \pm 0.03$ & $-0.02 \pm 0.03$ & $-0.02 \pm 0.03$ & \\
\hline 4 & $0.41 \pm 0.03$ & $0.15 \pm 0.03$ & $0.05 \pm 0.03$ & $0.00 \pm 0.03$ & $-0.02 \pm 0.03$ & $-0.02 \pm 0.03$ & & \\
\hline 5 & $0.39 \pm 0.03$ & $0.12 \pm 0.03$ & $0.00 \pm 0.03$ & $-0.02 \pm 0.03$ & $-0.01 \pm 0.03$ & & & \\
\hline 6 & $0.32 \pm 0.03$ & $0.03 \pm 0.03$ & $-0.03 \pm 0.03$ & $-0.04 \pm 0.03$ & & & & \\
\hline 7 & $0.23 \pm 0.03$ & $-0.02 \pm 0.03$ & $-0.05 \pm 0.03$ & & & & & \\
\hline 8 & $0.11 \pm 0.03$ & $-0.05 \pm 0.03$ & & & & & & \\
\hline 9 & $-0.04 \pm 0.03$ & & & & & & & \\
\hline
\end{tabular}

Table 4. Correlation coefficients $r_{i j}$ (7) for ECs created in liquid xenon by GQs of energy $555 \mathrm{MeV}$ at $E_{c o}=1 \mathrm{MeV}$.

\begin{tabular}{|l|l|l|l|l|l|l|l|l|}
\hline $\mathrm{i} / \mathrm{j}$ & 3 & 4 & 5 & 6 & 7 & 8 & 9 & 10 \\
\hline 2 & $0.53 \pm 0.02$ & $0.32 \pm 0.03$ & $0.22 \pm 0.03$ & $0.12 \pm 0.03$ & $0.07 \pm 0.03$ & $0.04 \pm 0.03$ & $0.01 \pm 0.03$ & $-0.10 \pm 0.03$ \\
\hline 3 & $0.57 \pm 0.02$ & $0.35 \pm 0.03$ & $0.21 \pm 0.03$ & $0.11 \pm 0.03$ & $0.04 \pm 0.03$ & $0.02 \pm 0.03$ & $-0.11 \pm 0.03$ & \\
\hline 4 & $0.56 \pm 0.02$ & $0.31 \pm 0.03$ & $0.17 \pm 0.03$ & $0.08 \pm 0.03$ & $0.02 \pm 0.03$ & $-0.09 \pm 0.03$ & & \\
\hline 5 & $0.52 \pm 0.02$ & $0.28 \pm 0.03$ & $0.12 \pm 0.03$ & $0.02 \pm 0.03$ & $-0.11 \pm 0.03$ & & & \\
\hline 6 & $0.46 \pm 0.03$ & $0.18 \pm 0.03$ & $-0.04 \pm 0.03$ & $-0.09 \pm 0.03$ & & & & \\
\hline 7 & $0.37 \pm 0.03$ & $0.08 \pm 0.03$ & $-0.09 \pm 0.03$ & & & & & \\
\hline 8 & $0.22 \pm 0.03$ & $-0.09 \pm 0.03$ & & & & & & \\
\hline 9 & $-0.09 \pm 0.03$ & & & & & & & \\
\hline
\end{tabular}

Table 5. The same as in Table 4 but for GQs of energy $2625 \mathrm{MeV}$ at $E_{c o}=1 \mathrm{MeV}$.

\section{References}

[1] Konrad Kleinknecht. Detectors for particle radiation. Cambridge University Press. London-New York-New Rochelle-Melbourne-Sydney, 1986; C.Grupen. Particle detectors. Cambridge University Press. 1996; Dan Green. The Physics of Particle Detectors. Cambridge University Press.2000; Review of Particle Physics. W.-M. Yao, et al., Journal of Physics G33, 1 (2006).

[2] B. Słowiński, Electron-photon showers produced by gamma quanta and electrons in dense amorphous media. Phys. Part. Nucl. 25 (2) March-April 1994.

[3] D.W.O. Rogers, I. Kawrakow, J.P. Seuntjens, B.R.B. Walters, E. Mainegra-Hing. NRCC Report PIRS-702(revB), 2005.

[4] GEANT code, CERN.

[5] E.V.Kuznetsov et al. Prib. Tekhn. Exper. (Russian), N.2, 170, 56-59. 